\title{
Approche du système de relations urbaines à travers l'analyse des flux de circulation routière : l'exemple de Lons-le-Saunier (Jura)
}

Understanding urban relations using road network flows : the case of Lons-leSaunier (Jura)

Überlegungen zum System städtischer Beziehungen mittels der Analyse von Strassen-verkerhrsströmen : das Beispeil von Lons-le-Saunier (Jura)

\section{Pascal Bérion}

\section{(2) OpenEdition}

\section{Journals}

Édition électronique

URL : http://journals.openedition.org/rge/4267

DOI : $10.4000 /$ rge.4267

ISSN : 2108-6478

Éditeur

Association des géographes de l'Est

Édition imprimée

Date de publication : 1 septembre 1999

ISSN : 0035-3213

\section{Référence électronique}

Pascal Bérion, « Approche du système de relations urbaines à travers l'analyse des flux de circulation routière : l'exemple de Lons-le-Saunier (Jura) », Revue Géographique de l'Est [En ligne], vol. 39 / 4 | 1999, mis en ligne le 12 août 2013, consulté le 08 septembre 2020. URL : http://journals.openedition.org/ rge/4267 ; DOI : https://doi.org/10.4000/rge.4267

Ce document a été généré automatiquement le 8 septembre 2020

Tous droits réservés 


\section{Approche du système de relations urbaines à travers l'analyse des flux de circulation routière : l'exemple de Lons-le-Saunier (Jura)}

Understanding urban relations using road network flows : the case of Lons-leSaunier (Jura)

Überlegungen zum System städtischer Beziehungen mittels der Analyse von Strassen-verkerhrsströmen : das Beispeil von Lons-le-Saunier (Jura)

Pascal Bérion

Préfecture du département du Jura, Lons-le-Saunier était, jusqu'au 2 juin 1998, dépourvue de desserte autoroutière. Les accès par ce type d'infrastructure étaient alors assurés à $40 \mathrm{~km}$ au Nord par l'entrée A 39-A 36 de Dole-Choisey, à $60 \mathrm{~km}$ à l'Ouest par l'A 6 à Chalon-sur-Saône et à $60 \mathrm{~km}$ au Sud par l'A 40 à Bourg-Viriat (fig. 1). Dans ce contexte, l'accessibilité routière du chef-lieu du Jura était effectuée par la RN 83 orientée nord-sud et la RN 78 orientée est-ouest et permettant les liaisons vallée de la Saône-Lons-le-Saunier et Lons-le-Saunier-Haute-Chaîne du Jura. 
Figure 1 : Environnement routier et urbain de Lons-le-Saunier.

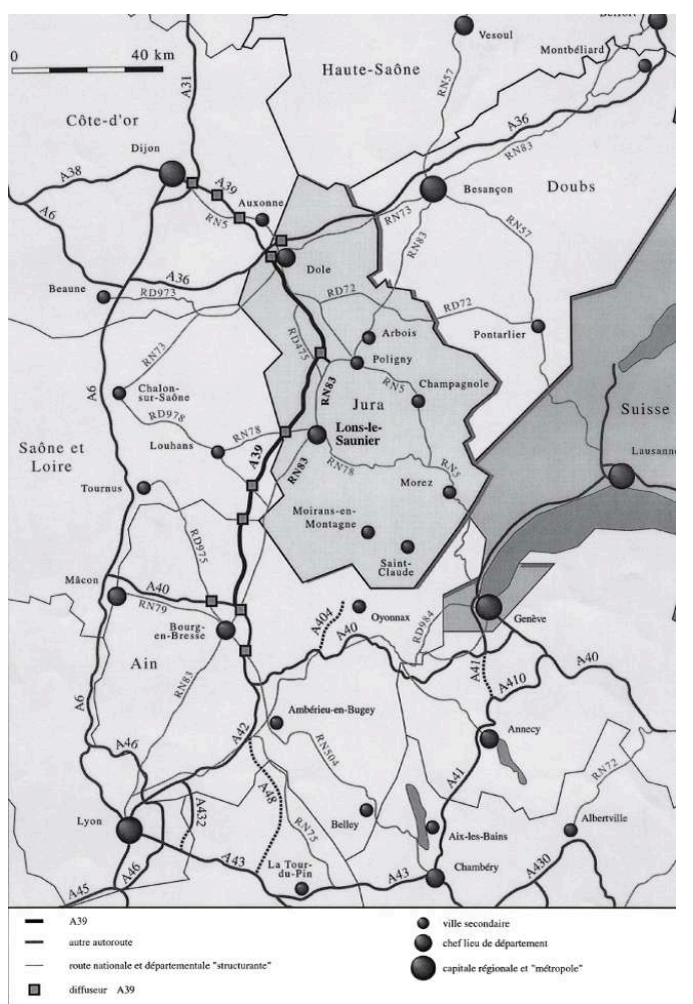

2 Les relations de la capitale jurassienne avec les espaces et les villes voisines sont, dans une certaine mesure, tributaires de cette organisation du réseau de transport routier. Avec la mise en service de l'autoroute A 39, il se produit une amélioration de l'offre de transport existante qui pourrait modifier les mobilités spatiales des usagers (D. Mathieu, en ce volume). Cependant, ces transformations devraient être spatialement différenciées: si l'autoroute ne présente aucun intérêt pour les déplacements de proximité, il n'en est pas de même pour les relations interurbaines pour lesquelles les gains d'accessibilité sont très inégaux. À terme, il est possible qu'une certaine recomposition affecte les aires d'influence et la hiérarchie du réseau urbain régional.

3 Au-delà, et en se référant aux travaux réalisés par le LET $^{1}$ pour la détection des effets $\mathrm{TGV}$, l'analyse de la transformation des flux, tant dans leur organisation géographique que dans leurs volumes et motivations, pourra aider à la lecture des évolutions socioéconomiques de l'agglomération lédonienne ${ }^{2}$, et à leur compréhension, au regard de l'ouverture de l'autoroute A 39. Dans le cadre de l'Observatoire A $39^{3}$, ces problématiques ont déjà fait l'objet de recherches conduites avec des angles d'approche différents (mobilité des Lédoniens, niveaux de service et hiérarchie urbaine). Les enquêtes de circulation viennent donc compléter et enrichir ce dispositif.

En termes de méthodes, rappelons que des postes d'interview ont été installés aux trois principales entrées de l'agglomération : RN 83 nord, RN 83 sud et RN 78 ouest (fig. 2). Les enquêtes réalisées en mai 1997 par le CETE de Lyon (avec l'appui d'étudiants bisontins) seront renouvelées à l'identique en 1999 et en 2003 pour déceler progressivement les éventuelles transformations. 
Figure 2 : Flux de véhicules légers émis et reçus par Lons-le-Saunier.

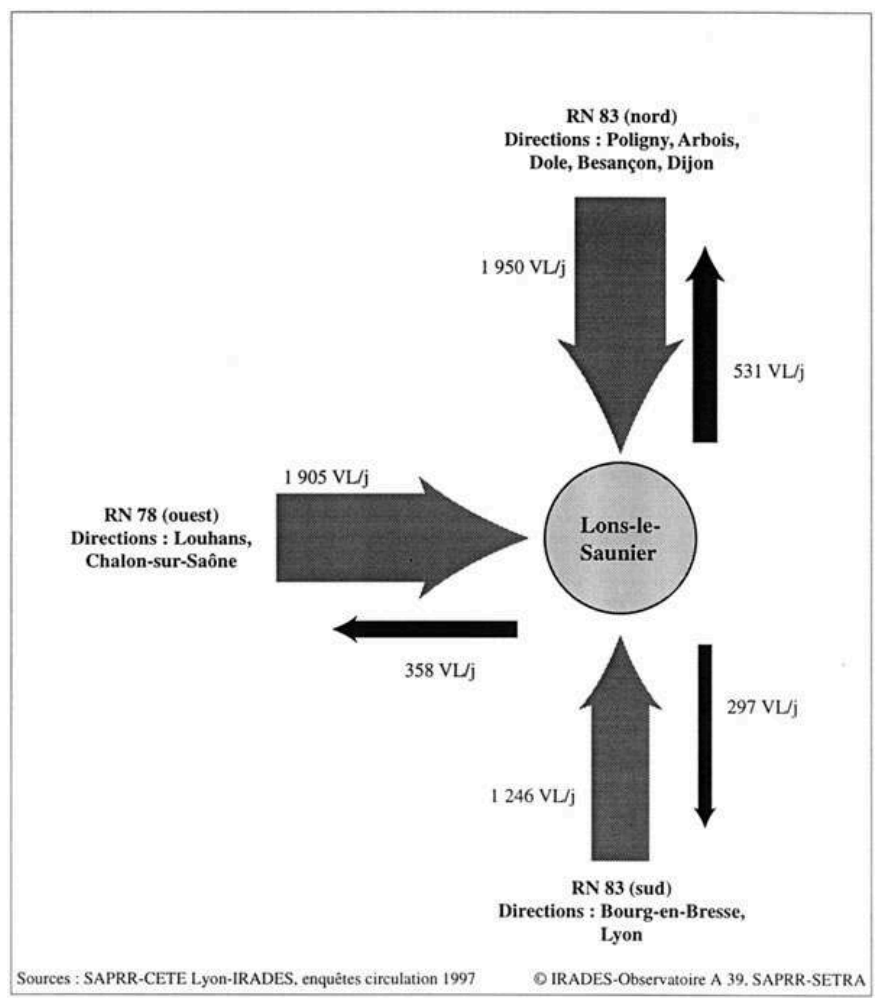

\section{Identification des flux enregistrés aux entrées de l'agglomération lédonienne}

$5 \mathrm{Au}$ total, et en valeurs redressées selon le coefficient de sondage horaire pendant la période d'enquête, l'agglomération lédonienne reçoit durant les 12 heures d'une journée moyenne en semaine et par ses trois principales entrées, 6700 véhicules à savoir 6300 véhicules légers (VL) et 400 poids-lourds (PL) (tableau 1).

Tableau 1 : Composition des flux observés aux entrées de Lons-le-Saunier

\begin{tabular}{|l|c|c|c|c|c|c|}
\hline & \multicolumn{3}{|c|}{ Flux total } & Flux à destination de Lons-le-Saunier \\
\hline Entrées & VL & PL & Total & VL & PL & Total \\
\hline RN 83 Nord & 4537 & 879 & 5416 & 2481 & 175 & 2656 \\
\hline RN 83 Sud & 2880 & 718 & 3598 & 1543 & 122 & 1665 \\
\hline RN 78 Ouest & 2768 & 236 & 3004 & 2263 & 90 & 2353 \\
\hline Total & 10185 & 1833 & 12018 & 6287 & 387 & 6674 \\
\hline Source : SAPRR-CETE Lyon-IRADES, enquêtes circulations 1997, données redressées période d'enquête. \\
\hline
\end{tabular}

6 Les enquêtes révèlent que l'agglomération organise les flux identifiés à ses entrées, puisque $55,5 \%$ du flux total lui est destiné. Toutefois, cet indicateur met en évidence la présence d'une incontestable fonction de transit. Il faut cependant souligner qu'il existe une nette opposition entre le trafic VL et le trafic PL. Ainsi, Lons-le-Saunier capture $62 \%$ du flux de VL contre seulement $21 \%$ des PL (tableau 2). 
Tableau 2 : Taux de destination à Lons-le-Saunier.

\begin{tabular}{|l|c|c|c|}
\hline & \multicolumn{3}{|c|}{$\begin{array}{c}\text { Taux de destination } \\
\text { à Lons-le-Saunier }\end{array}$} \\
\hline EntréesVL & PL & Ensemble & du flux \\
\hline RN 83 Nord & 54,7 & 20,0 & 49,0 \\
\hline RN 83 Sud & 53,5 & 17,0 & 46,3 \\
\hline RN 78 Ouest & 81,7 & 38,0 & 78,3 \\
\hline Total & 61,7 & 21,0 & 55,5 \\
\hline $\begin{array}{l}\text { Source: SAPRR-CETE Lyon-IRADES, enquêtes circulations } \\
\text { 1997, données redressées période d'enquête. }\end{array}$ \\
\hline
\end{tabular}

7 De ce fait, le trafic de transit et le trafic à destination de Lons-le-Saunier sont de natures très dissemblables. $\mathrm{Si}$, en moyenne, on observe aux entrées des flux se composant de $85 \%$ de VL et $15 \%$ de PL, on retrouve à destination du chef lieu du Jura $94 \%$ de VL et $6 \%$ de PL. Ainsi, comme il n'existe toujours pas de voie de déviation de Lons-le-Saunier, l'agglomération subit un flux de transit important, d'environ 3500 véhicules pour les trois entrées prises en compte dont $1450 \mathrm{PL}$ ( $40 \%$ du transit total). On peut espérer que l'A 39 soulagera l'agglomération d'une partie de ce flux puisque les chauffeurs de PL interrogés sur ce point durant les enquêtes ont déclaré, pour $44 \%$ d'entre eux, qu'ils emprunteront dorénavant l'A 39. Cependant, $24 \%$ ne se sont pas prononcés et $32 \%$ affirment ne pas avoir intérêt à l'utiliser.

Par ailleurs, de sensibles différences s'observent selon les postes. Les entrées nord et sud de la RN 83 offrent des profils voisins. Moins de $50 \%$ du flux est dirigé vers Lons-leSaunier ( $49 \%$ au nord et $46 \%$ au sud). Inversement, pour l'entrée ouest le transit est faible puisque $78 \%$ du flux a pour destination Lons-le-Saunier ( $82 \%$ des VL et $38 \%$ des PL). De plus cette entrée est peu utilisée par les PL qui ne correspondent qu'à $8 \%$ du flux total contre $16 \%$ et $20 \%$ aux entrées nord et sud. Toutefois, il faut relativiser quelque peu la portée de ces observations, car le poste d'enquête a été situé, pour des raisons techniques, au plus près de l'agglomération, là où les flux des VL sur courte distance sont les plus importants.

Pour les flux destinés à Lons-le-Saunier qui, seuls, nous intéressent ici, on observe de nettes différences, en nature et en volume, entre les postes d'observation.

L'accès nord est le plus chargé : avec 2650 véhicules il se place devant l'accès ouest (2 350 véhicules) et l'entrée sud (1 660 véhicules). Cette organisation s'explique sans doute par des facteurs d'ordre géographique. Au nord, dans un rayon de 20 à $40 \mathrm{~km}$, la densité du peuplement liée à la présence de villes (Arbois, Dole et Poligny) favorise la multiplication des déplacements. Vers le sud l'absence de villes proches - la première, Bourg-en-Bresse, étant à $60 \mathrm{~km}$ - réduit logiquement l'ampleur des échanges. Le poste ouest offre une situation intermédiaire où l'isolement de la plaine de Bresse fait de 
Lons-le-Saunier un site de fréquentation privilégié et ce, malgré l'attraction de l'agglomération de Louhans (10 000 habitants), distante seulement de $20 \mathrm{~km}$.

11 La prise en compte des lieux de résidence des enquêtés conduit à identifier deux groupes ; d'une part ceux qui n'habitent pas dans l'agglomération et qui se dirigent vers celle-ci pour un motif spécifique, d'autre part des Lédoniens qui ont effectué un déplacement en dehors de l'agglomération et qui sont interrogés sur le trajet de retour.

Les chiffres globaux font apparaître une nette dissymétrie puisque le flux des Lédoniens vers l'extérieur est très nettement inférieur au flux inverse, le rapport entre les deux étant schématiquement de 1 à 4 pour les trois postes d'observation. Il faut cependant noter que pour l'entrée ouest, la part des Lédoniens dans le trafic hors transit est inférieure à $16 \%$. Cela suggère que, par rapport au problème de la mobilité, la RN 78 n'assure pas exactement la même fonction que la RN 83 .

La prise en compte des motifs de déplacements permet d'apporter quelques éléments d'explications à ces constatations.

\section{Des flux dirigés vers Lons-le-Saunier, régis par la dualité centre-périphérie}

14 Pour les personnes habitant à l'extérieur de l'agglomération et se rendant à Lons-leSaunier, l'analyse des origines géographiques précises et des motifs des déplacements permet de replacer l'essentiel de ces flux dans une problématique de relation centrepériphérie (tableau 3).

Tableau 3 : Composition du flux de VL dirigé vers Lons-le-Saunier.

\begin{tabular}{|l|c|c|c|c|c|c|}
\hline \multicolumn{1}{|c|}{ Entrées } & Total & Dest. Lons & $\begin{array}{c}\text { dont } \\
\text { Lédoniens }\end{array}$ & $\begin{array}{c}\text { dont } \\
\text { externes }\end{array}$ & $\%$ Lédoniens & $\%$ externes \\
\hline RN 83 N & 4537 & 2481 & 531 & 1950 & 21,4 & 78,6 \\
\hline RN 83 S & 2880 & 1543 & 297 & 1246 & 19,4 & 80,8 \\
\hline RN 78 O & 2768 & 2263 & 358 & 1905 & 15,8 & 84,2 \\
\hline Total & 10185 & 6287 & 1186 & 5101 & 18,8 & 81,2 \\
\hline Source : SAPRR-CETE Lyon-IRADES, enquêtes circulations 1997, données redressées période d'enquête.
\end{tabular}

En premier lieu, on note en effet que $57 \%$ des déplacements se font sur des distances inférieures à $20 \mathrm{~km}$ : les populations périurbaines et rurbaines constituent donc l'essentiel du flux se rendant à Lons-le-Saunier. La prise en compte du motif permet d'éclairer la nature de ces relations de proximité. Les " raisons personnelles ", qui dans la nomenclature employée pour l'enquête recouvrent la chalandise, les visites à des parents ou amis, ainsi que les diverses démarches administratives, arrivent en tête (1 200 mouvements), suivies de très près par le motif « déplacement quotidien de travail » (1 070), c'est-à-dire la relation domicile-travail. Le poids écrasant de ces deux motifs de déplacements pour les relations à courte distance, met bien en évidence l'attraction de Lons-le-Saunier sur les espaces périphériques, tant par ses fonctions commerciales et de services, que par le pôle d'emplois qu'elle constitue.

Les déplacements effectués par des résidents des unités urbaines environnantes en direction de Lons-le-Saunier sont, en volume, moins importants ( $43 \%$ ) que les relations 
de proximité. Leur analyse fournit des résultats qui confirment certaines observations réalisées par ailleurs par l'Observatoire avec d'autres méthodes de mesures ${ }^{4}$.

En fonction de la taille des agglomérations et de leur distance à Lons-le-Saunier, trois types de situations peuvent être mis en évidence (tableau 4).

Tableau 4 : Nature des déplacements émis depuis les villes proches (Poligny, Louhans, Arbois)

\begin{tabular}{|l|c|c|}
\hline & $\begin{array}{c}\text { Nb } \\
\text { déplacements }\end{array}$ & en $\%$ \\
\hline Motif personnel & 280 & 40,0 \\
\hline Motif professionnel & 211 & 30,0 \\
\hline Migration alternante & 166 & 23,5 \\
\hline Autres motifs & 48 & 6,5 \\
\hline Total & 705 & \\
\hline $\begin{array}{l}\text { Source: SAPRR-CETE Lyon-IRADES, enquêtes circulations } \\
\text { 1997, données redressées période d'enquête. }\end{array}$ \\
\hline
\end{tabular}

18 En premier lieu, les liens avec les petites villes proches de la capitale jurassienne s'individualisent nettement, tant dans les volumes émis que par les motifs des déplacements. Il en est ainsi et par ordre décroissant, pour Poligny, Louhans et Arbois, villes situées à une vingtaine de kilomètres de Lons-le-Saunier. Ici deux motifs dominent, les «raisons personnelles" et les "raisons professionnelles». Le motif «déplacement quotidien de travail » n'arrive qu'en troisième position, et sauf pour Poligny, il est relégué très loin des précédents. Il est clair qu'en termes de centre d'emploi, Lons-le-Saunier n'a qu'une influence relativement modeste sur les petites villes voisines, alors qu'elle reste très attractive par ses fonctions de centre tertiaire, tant pour les particuliers que pour les milieux économiques.

En deuxième lieu, il semble bien exister entre Dole et Besançon d'une part, et Lons-leSaunier d'autre part, un lien d'une nature très spécifique qui tient à l'appartenance de ces trois villes à la même entité administrative régionale. Dole, dont la taille est équivalente à celle de Lons-le-Saunier, dirige vers cette dernière 250 mouvements ainsi structurés : motifs professionnels $(57,5 \%)$, motifs personnels $(20 \%)$ et migrations alternantes (16\%). Les flux en provenance de Besançon sont plus faibles (160 mouvements) sans doute en fonction de l'éloignement, mais la répartition suivant le motif reste très voisine avec $57,5 \%$ de déplacements professionnels : on note toutefois une baisse des motifs personnels (11\%), les Bisontins ayant sur place un équipement en commerces et services bien supérieur à celui de la capitale jurassienne.

Enfin, la fréquentation de Lons-le-Saunier par les Burgiens et Chalonnais d'une part, par les Dijonnais et Lyonnais d'autre part, suscite quelques interrogations. Situées à 60-70 km de l'agglomération lédonienne, Bourg-en-Bresse et Chalon-sur-Saône ont un poids démographique plus important (60000 habitants contre 25000 ). Pourtant, malgré leur taille et leur proximité, le lien est faible (80 et 60 mouvements) et 
s'explique sans doute par l'appartenance de ces deux villes à d'autres entités administratives et d'autres réseaux de sociabilité.

Pour Dijon et Lyon, et malgré un éloignement plus grand, les volumes des flux restent du même ordre, mais le poids démographique de l'agglomération lyonnaise lui assure une certaine prééminence sur la capitale bourguignonne (110 mouvements contre 60).

Concernant les poids-lourds, l'enquête apporte des informations sur les originesdestinations des flux, la nature des marchandises et les tonnages transportés. L'analyse des livraisons à Lons-le-Saunier permet une approche de l'organisation spatiale des approvisionnements. Ainsi, sur 3063 tonnes livrées, $51 \%$ ont été chargées à moins de $80 \mathrm{~km}$ de Lons-le-Saunier : elles représentent les échanges de proximité en provenance $\mathrm{du}$ réseau urbain proche (Bourg-en-Bresse, Dole, Arbois, Poligny et Louhans). $27 \%$ arrivent des villes un peu plus éloignées (Lyon, Besançon, Dijon, Chalon-sur-Saône, Mâcon...), alors que les livraisons en provenance d'autres régions françaises ou d'autres pays, représentent encore $22 \%$ du total.

Les informations sur la nature et le volume des produits livrés renseignent d'une certaine manière sur l'activité économique de l'agglomération. Les deux rubriques les mieux représentées sont les biens manufacturés ( $25 \%$ du tonnage réceptionné) et les denrées alimentaires pour la vente $(22 \%)$. Globalement on peut estimer que près de $50 \%$ du tonnage livré est constitué de produits finis directement commercialisables. Les matières premières ou biens intermédiaires destinés à des fonctions de production constituent environ un tiers des livraisons, les $20 \%$ restants relevant d'usages variés (produits pétroliers) ou mal déterminés.

Faute d'éléments de comparaison, il est difficile de tirer des conclusions à partir des indicateurs fournis par le trafic poids-lourds. Mais ils serviront de référence pour les enquêtes qui seront réalisées en 1999 et 2003. La mise en relation des différents résultats devrait permettre de tirer des enseignements sur l'évolution économique de l'agglomération lédonienne.

\section{Des flux émis par Lons-le-Saunier qui confirment la prédominance de liens administratifs et sociaux}

25 L'analyse des flux émis à partir de Lons-le-Saunier apporte une information moins riche que celle de la situation précédente. En effet, le nombre d'enquêtés est peu important, ce qui n'autorise pas une exploitation statistique très poussée, en particulier pour les poids-lourds.

Pour les véhicules légers, les deux destinations principales sont Besançon et Dole. Le volume émis est équivalent, dans les deux cas, une centaine de déplacements. Toutefois, leur structure montre quelques différences. Vers Besançon, $58 \%$ des mouvements sont effectués pour des raisons professionnelles et $28 \%$ pour motifs personnels. Vers Dole $53 \%$ sont d'ordre professionnel, mais $26 \%$ correspondent à des migrations alternantes (contre $10 \%$ à Besançon), ce qui s'explique par la plus grande proximité de Dole ( $40 \mathrm{~km}$ contre $80 \mathrm{~km})$.

27 La faiblesse du motif personnel (15\%) pour les déplacements des Lédoniens en direction de Dole tient en partie au fait que cette dernière n'assure pas de fonctions administratives majeures: pour le département du Jura, celles-ci sont assurées par Lons-le-Saunier, elles expliquent d'ailleurs un flux inverse soutenu. Des raisons 
similaires expliquent l'importance des déplacements pour motifs personnels en direction de Besançon, qui attire par ses fonctions de capitale régionale.

Par ailleurs, il est probable que les modalités de réalisation de l'enquête conduisent à sous-estimer la part des motifs personnels : effet les interviews se sont terminés à 19 heures, alors que les retours de fin de journée vers Lons-le-Saunier sont loin d'être terminés.

Les déplacements des Lédoniens vers les petites villes proches sont relativement nombreux (230 vers Poligny, Louhans et Arbois). Les motifs sont d'abord d'ordre professionnel (42\%) mais les migrations alternantes tiennent une place non négligeable (30\%). La capitale jurassienne aurait-elle une attractivité résidentielle plus grande que les petites cités voisines?

Il faut enfin noter que les relations des Lédoniens en direction des ensembles urbains plus éloignés (Lyon et Dijon) sont trop peu nombreuses pour que leur analyse soit crédible.

\section{Conclusion}

31 Les informations obtenues par l'enquête de circulation routière révèlent qu'avant l'ouverture de l'autoroute A 39, le système relationnel de l'agglomération est peu ouvert sur l'extérieur. Les relations se font en priorité en direction des périphéries immédiates et des petites villes proches pour lesquelles Lons-le-Saunier assure une incontestable fonction de centralité.

Quant aux échanges avec les entités urbaines plus éloignées et de rang hiérarchique plus élevé, les destinations privilégiées sont Dole et Besançon, villes à la fois peu éloignées et structurellement associées dans les mêmes entités administratives (département, région). Dans un tel système, la mise en service de l'autoroute A 39 ne devrait pas apporter de changements fondamentaux dans la structuration des flux. Pourtant, l'ouverture de l'espace lédonien en direction de Dijon et surtout de Lyon pourrait renforcer des relations aujourd'hui très occasionnelles et intégrer un peu plus la préfecture du Jura dans l'aire d'influence de la métropole rhodanienne. À l'inverse, on devrait assister à un affaiblissement des relations avec Besançon dont l'accessibilité ne sera pas améliorée sensiblement.

\section{BIBLIOGRAPHIE}

Bérion P. (1996). - Grandes infrastructures de transport et aménagement du territoire : méthodologie et mise en place de l'Observatoire des effets territoriaux de l'autoroute A 39, Thèse de doctorat en géographie, Université de Franche-Comté, Besançon, 608 pages.

Bernadet J.L. et al. (1990). - Autoroutes interurbaines et agglomérations, LET METT, Lyon, 137 pages. 
Biehl D. (1991). - The role of infrastructure in regional development, in Wickerman R.-W., Infrastructure and regional development, Pion, London, pp. 9-35.

Bonnafous A., Plassard F., Vulin B. et al. (1993). - Circuler demain, DATAR, Éditions de l'Aube, Paris, 191 pages.

CETE de Lyon (1998). - Bilan LOTI transport point zéro 1997, SAPRR, CETE de Lyon, 77 pages.

Claisse G., Duchier D. (1993). - Des observatoires d'effets TGV : réflexions méthodologiques, Laboratoire d'Économie des Transports, Colloque villes et TGV, Lyon, décembre 1993, 16 pages.

Heddebaut O. (sous la dir. de) (1997). - Grandes infrastructures de transport et territoires, Actes du colloque des 8 et 9 juin 1995, INRETS Actes $n^{\circ}$ 60, Lille, 231 pages.

Klein O., Claisse G. (1997). - Le TGV-Atlantique : entre récession et concurrence, Laboratoire d'Économie des Transports, Études et Recherches, Lyon, 163 pages

Varlet J. (dir.) (1997). - Autoroutes, économie, territoires, Actes du colloque de mai 1997, ClermontFerrand, CERAMAC, 423 pages.

\section{NOTES}

1. LET : Laboratoire d'Économie des Transports, Université Lumière, Lyon 2.

2. Par agglomération lédonienne, il est entendu, unité urbaine de Lons-le-Saunier définie par l'INSEE.

3. L'Observatoire des effets de l'autoroute A 39 est une procédure scientifique expérimentale d'identification et d'interprétation des effets d'une grande infrastructure de transport financée par la SAPRR (Société des Autoroutes Paris-Rhin-Rhône) et le ministère de l'Équipement (Service d'Études Techniques des Routes et Autoroutes).

4. Rayonnement des Villes : état initial. P. Berion, M. Le-Berre, D. Mathieu, Observatoire A 39, 1995.

\section{RÉSUMÉS}

Derrière toute analyse de la mobilité dans l'espace géographique, le chercheur tente de déceler les structures et l'organisation territoriale qui génèrent ces déplacements. Dans le cadre des recherches de l'observatoire des effets économiques de l'autoroute A 39, cette approche est conduite pour aider à la compréhension des transformations territoriales que peut susciter la mise en service d'une nouvelle liaison autoroutière. L'approche ici présentée est relative au système de déplacements par mode routier structuré par l'agglomération de Lons-le-Saunier. Elle dresse un état initial de l'organisation spatiale et de la nature des flux routiers avant la mise en service de l'autoroute A 39. Elle révèle que si les modèles gravitaires sont de précieux guides pour l'interprétation des phénomènes de mobilité, les structures spatiales et territoriales sont aussi de puissants catalyseurs de déplacement qui se jouent des contraintes de distance.

Underpinning alla analysis of spatial mobility is the need to discover the structure and territorial organisation which generate transfers. From the point of view of research into the economic 
effects of motorway A39, this approach helps the comprenhension of territorial changes which can arise from the introduction of a new motorway link. Our approach tries to study the effects of the town of Lons-le-Saunier on road network flows. We establish the initial spatial organisation and pattern of the road network flows prior to the installation of the A39 motorway. Whe show that gravity models are invaluable guides for interpreting mobility; spatial and territorial structures are also powerful catalysts of flow which mitigate the constraints of distance.

Hinter jeder Analyse der Mobilität in geographischen Raum sucht der Forscher die Strukturen und die Raumorganisation zu entdecken, welche diese Verlagerungen erzeugen. Im Rahmen der Forschungen des Observatoriums über die wirtschaftlichen Auswirkungen der Autobahn A39 möchten diese Überlegungen dem Verständnis für die räumlichen Veränderungen dienen, welche die Eröffnung einer neuen Autobahnverbindung hervorrufen kann. Vorliegender Beitrag bezieht sich auf die Verlagerungen im Strassenverkehr, strukturiert durch die Agglomeration von Lons-le-Saunier. Es wird ein Initialzustand der Raumorganisation und der Strassenverkehrsströme vor Eröffnung der Autobahn A39 vorgestellt. Es wird deutlich, dass die Gravitationsmodelle wertvolle Hilfe zur Interpretation der Mobilitätsphänomene bieten, dass aber auch die territorialen Raumstrukturen mächtige Katalysatoren der Verlagerung sind, ungeachtet der Zwänge der Entfernung.

\section{INDEX}

Schlüsselwörter : Autobahn, Einflussbereich der Städte, Erreichbarkeit, Mobilität

Keywords : accessibility, influence of towns, mobility, motorway

Mots-clés : accessibilité, autoroute, mobilité, rayonnement des villes

\section{AUTEUR}

\section{PASCAL BÉRION}

IRADES@THEMA-UPRESA 6049 du CNRS - Université de Franche-Comté - 32, rue Mégevand 25030 Besançon Cedex / Observatoire des effets économiques de l'autoroute A 39, SAPRR-SETRA, avec la collaboration du CETE de Lyon 